\title{
Discovery of highly excited long-lived isomers in neutron-rich hafnium and tantalum isotopes through direct mass measurements
}

\author{
M.W. Reed, ${ }^{1, *}$ I.J. Cullen, ${ }^{1}$ P.M. Walker, ${ }^{1}$ Yu.A. Litvinov ${ }^{2}{ }^{2}$ K. Blaum,${ }^{2}$ F. \\ Bosch, ${ }^{3}$ C. Brandau, ${ }^{3,4}$ J.J. Carroll, ${ }^{5}$ D.M. Cullen, ${ }^{6}$ A.Y. Deo, ${ }^{1}$ B. Detwiller,${ }^{5}$ C. \\ Dimopoulou, ${ }^{3}$ G.D. Dracoulis, ${ }^{7}$ F. Farinon, ${ }^{3}$ H. Geissel, ${ }^{3,8}$ E. Haettner,${ }^{8}$ M. Heil,${ }^{3}$ \\ R.S. Kempley, ${ }^{1}$ R. Knöbel, ${ }^{3}$ C. Kozhuharov ${ }^{3}$ J. Kurcewicz, ${ }^{3}$ N. Kuzminchuk, ${ }^{3}$ S. \\ Litvinov, ${ }^{3}$ Z. Liu,${ }^{9}$ R. Mao, ${ }^{10}$ C. Nociforo,${ }^{3}$ F. Nolden, ${ }^{3}$ W.R. Plass,${ }^{8}$ A. Prochazka,${ }^{3}$ \\ C. Scheidenberger, ${ }^{3,8}$ M. Steck, ${ }^{3}$ Th. Stöhlker, ${ }^{3,11}$ B. Sun, ${ }^{3}$ T.P.D. Swan, ${ }^{1}$ G.
} Trees,${ }^{5}$ H. Weick, ${ }^{3}$ N. Winckler, ${ }^{2,3}$ M. Winkler, ${ }^{3}$ P.J. Woods,${ }^{9}$ and T. Yamaguchi ${ }^{12}$

${ }^{1}$ Department of Physics, University of Surrey, Guildford, Surrey GU2 7XH, United Kingdom ${ }^{2}$ Max-Planck-Institut für Kernphysik,

Saupfercheckweg 1, 69117 Heidelberg, Germany ${ }^{3}$ GSI Helmholtzzentrum für Schwerionenforschung,

Planckstraße 1, 64291 Darmstadt, Germany

${ }_{4}^{4}$ Physik-Department E12, Technische Universität München, 85748 Garching, Germany

${ }^{5}$ Youngstown State University, One University Plaza, Youngstown, Ohio 44555, USA

${ }^{6}$ Schuster Laboratory, University of Manchester, Manchester M13 9PL, United Kingdom

${ }^{7}$ Department of Nuclear Physics, R.S.P.E., Australian National University, Canberra ACT 0200, Australia

${ }^{8}$ II Physikalisches Institut, Justus-Liebig-Universität Gießen, 35392 Gießen, Germany

${ }^{9}$ School of Physics and Astronomy, University of Edinburgh, Edinburgh EH9 3JZ, United Kingdom

${ }^{10}$ Institute of Modern Physics, Chinese Academy of Sciences, Lanzhou 730000, PR China ${ }^{11}$ Physikalisches Institut, Universität Heidelberg, Philosophenweg 12, 69120 Heidelberg, Germany ${ }^{12}$ Graduate School of Science and Engineering, Saitama University, Saitama 338-8570, Japan 
(Dated: August 31, 2010)

\begin{abstract}
A study of cooled ${ }^{197} \mathrm{Au}$ projectile-fragmentation products has been performed with a storage ring. This has enabled metastable nuclear excitations with energies up to $3 \mathrm{MeV}$, and half-lives extending to minutes or longer, to be identified in the neutron-rich nuclides ${ }^{183,184,186} \mathrm{Hf}$ and ${ }^{186,187} \mathrm{Ta}$. The results support the prediction of a strongly favored isomer region near neutron number 116.
\end{abstract}

PACS numbers: 21.10.-k, 25.70.Mn, 27.70.+q, 29.20.Dh

*Electronic address: m.w.reed@surrey.ac.uk 
Major developments in storage-ring techniques mean that the mass of an individual ion can now be measured with sufficient accuracy to differentiate not only between nuclear isobars with the same mass number, $A=N+Z$, but also between a nuclear ground state and a metastable excited state, known as an isomer [1]. Isomers are found with a wide range of half-lives, from less than a nanosecond to more than the age of the Universe, and can have excitation energies exceeding $10 \mathrm{MeV}$. Ever since the existence of isomers was first postulated by Soddy [2], the relationship between isomers and their ground states has not only provided basic nuclear-structure information, but has also been a source of conjecture and controversy, especially with regard to an isomer's ability to store and release energy [3]. Now, with the research frontier addressing the limits of nuclear binding, there is a realization that highly excited $(>1 \mathrm{MeV})$ isomers with broken-nucleon-pair configurations can confer extra stability [4], at least in the sense that extended half-lives may be manifest. This is an important experimental issue since, with some of the most sensitive apparatus, longer half-lives (>1 minute) become harder to observe. Such a problem may hinder, for example, the identification of the "island of stability" of superheavy nuclei [5].

There is a long-standing prediction [6] of an isomer-rich landscape for deformed neutronrich $Z \approx 72$ (hafnium) nuclides, which has been supported by detailed model calculations $[1,7]$. Nevertheless, this domain of heavy, neutron-rich nuclei remains largely unexplored. Here, the nucleon numbers $Z=72$ and $N=116$, with eight neutrons more than the heaviest stable isotope, are predicted [8] to lead to optimum conditions for the formation of a highspin isomer in ${ }^{188} \mathrm{Hf}$. This would have two broken nucleon pairs, i.e. four quasiparticles, with $K^{\pi}=18^{+}$(where $K$ is the angular momentum projection on the nuclear symmetry axis) and an excitation energy of about $2.3 \mathrm{MeV}$. Such a state is analogous to the renowned $K^{\pi}=16^{+}$, $2.4 \mathrm{MeV}, 31$-year isomer in ${ }^{178} \mathrm{Hf}$ [9] whose combination of high spin, high excitation energy and long half-life is so-far unique, leading to unusual experimental possibilities. For example, attempts have been made to release the ${ }^{178} \mathrm{Hf}$ stored isomer energy by x-ray stimulation [10]. Although the initial positive indications have not been substantiated [11, 12], other isomers of similar character might yet provide the necessary conditions. An important related issue is that of isomer photo-destruction in astrophysical environments where nucleosynthesis occurs [13], and a better understanding is needed of long-lived isomers that lie away from the line of $\beta$-stable nuclides.

Experimentally, steady progress with the discovery of new isomers in the neutron-rich $Z \approx$ 
72 region has been made with deep-inelastic [14-16] and projectile-fragmentation [17, 18] reactions. Projectile fragmentation seems to offer the best prospects to extend well into the neutron-rich domain, but the ability to identify excited states in the exotic products has depended upon establishing time correlations between the identified fragments and their subsequent radioactive decays. This correlation becomes non-viable for half-lives longer than a few milliseconds when $\gamma$-rays are the detected radiation, extending to tens of seconds when $\beta$ particles [19] or conversion electrons [20] are observed. However, these techniques are insensitive to the predicted longer-lived isomers. The present letter now reports on the successful application of an alternative detection methodology with no half-life upper limit for isomer identification, whereby the projectile-like fragments are injected into a storage ring and their mass measured. In this way, new long-lived hafnium and tantalum isomers have been discovered.

The region of heavy, neutron-rich nuclei was accessed by the use of projectile fragmentation. The primary beam of ${ }^{197} \mathrm{Au}$ with intensity up to $5 \cdot 10^{9}$ particles/cycle was accelerated by the UNILAC-SIS facility at GSI to energies of 478-492 A-MeV (dependent on the fragment setting) and was incident on the production target placed in front of the fragment separator (FRS) [21]. The target was $1035 \mathrm{mg} / \mathrm{cm}^{2}{ }^{9} \mathrm{Be}$ with a $221 \mathrm{mg} / \mathrm{cm}^{2}$ niobium backing for more efficient electron stripping. The highly charged reaction products, frequently bare ions without atomic electrons, were separated with the FRS and injected into the experimental storage ring (ESR) [22]. A $200 \mu \mathrm{m}$ aluminum foil installed at the middle focal plane of the FRS gave different energy loss, $\Delta \mathrm{E}$, for fragments with different atomic numbers $\left(\Delta \mathrm{E} \sim Z^{2}\right)$. Thus exotic fragments of interest could be effectively separated from abundant, less-exotic fragments and from the primary beam, which would otherwise be present in several atomic charge states. More details of the separation modes of the FRS can be found in ref. [21].

The ions injected into the ESR were stochastically and electron cooled. The stochastic cooling operates at a fixed fragment energy of $400 \mathrm{~A} \cdot \mathrm{MeV}$ and therefore the energy of the primary beam was adjusted so that the investigated fragments had this energy after the aluminum foil. The mean circumference of the ESR is $108.4 \mathrm{~m}$ and the ions circulate with a frequency of about $2 \mathrm{MHz}$. The revolution frequencies of electron-cooled ions depend (to first order) only on their mass-to-charge ratio [23]. Time-resolved Schottky mass spectrometry [24] has been applied to measure the precise revolution frequencies and intensities of the stored ions. The frequencies are determined by a fast Fourier transform (FFT) of the 
signals induced by circulating ions on the dedicated Schottky pick-up plates installed inside the ring aperture. Typically the $30^{t h}$ harmonic of the revolution frequency is analyzed. For more details the reader is referred to refs. [23, 24]. For the present purposes, ions were injected into the ESR once per minute. The on-line monitoring of the experiment was done with a real-time spectrum analyzer (RSA) providing FFT spectra with a narrow bandwidth, while the data were also continuously digitized and stored for the off-line, broad-band FFT analysis. On some occasions, when a candidate isomer was observed in the RSA, the next injection was manually delayed until the candidate isomer had decayed or was otherwise lost from the ring. This enabled longer half-lives to be evaluated.

Regarding the identification of new isomers, there could in general be an ambiguity between a potential isomer and a less-bound isobar in its ground state present in the same atomic charge state. However, by choosing to observe bare $Z=72$ hafnium ions, for instance, the less-bound isobar of $Z=71$ lutetium was rigorously excluded. This provided a powerful and highly sensitive technique for the identification of long-lived isomers and the determination of their excitation energies, with no half-life upper-limit. Following the earlier identification of a low-lying $(0.1 \mathrm{MeV})$ isomer in ${ }^{125} \mathrm{Ce}[25]$, the present letter reports the first successful use of the storage-ring technique for the discovery of highly excited, long-lived isomers.

A selection of spectra from different injections is shown in Fig. 1, where the isomers can be clearly seen. Each spectrum represents data integrated over a period of ten seconds, which is sufficient to establish single-ion identifications. For the $A=184$ isobars, the ${ }^{184} \mathrm{Ta}^{72+}$ ground state provides a convenient reference frequency, which has been used in the off-line analysis to make corrections for small frequency drifts arising from slow changes in the power supplies of the magnets and the electron cooler. Correspondingly, for the $A=187$ isobars, the ${ }^{187} \mathrm{~W}^{73+}$ and ${ }^{187} \mathrm{Re}^{73+}$ ground states provide reference frequencies. From the analysis of many spectra of this kind, isomer excitation energies (corresponding to the frequency differences in Fig. 1) could be determined with an uncertainty down to $\pm 10 \mathrm{keV}$. The detailed results are given in Table I.

The data for ${ }^{184} \mathrm{Hf}$ are discussed first. Krumbholz et al. [14] had already assigned a long-lived $\gamma$-decaying $K^{\pi}=8^{-}$isomer at $1.272(1) \mathrm{MeV}$. Our confirmation of an isomer at 1.264(10) MeV eliminates the residual possibility that the long lifetime originally observed could be due to $\beta$ decay from a ${ }^{184} \mathrm{Lu}$ parent. This is ruled out by the restriction to a 
$72+$ charge state. Furthermore, a second ${ }^{184} \mathrm{Hf}$ isomer (see Fig. 1) is now observed, with an excitation energy of 2.477(10) MeV. On five occasions, following on-line observation of a single bare ion of this isomer, the subsequent injection into the ESR was blocked, and the survival of the ion was monitored. In four cases, the ion was lost from the ESR after some minutes (4, 9, 11 and 18 minutes) and in the other case it survived for 27 minutes before the following injection was made. The principal loss mode for stable bare ions is electron pick-up from the cooler, and the estimated average time for this to happen to bare hafnium ions is 50(9) minutes, for the applied cooler current of $250 \mathrm{~mA}$. This estimate was obtained by observing the loss of bare ions of ${ }^{194} \mathrm{Ir}$ during the same experiment, and scaling the loss rate with $Z^{2}$. Accordingly, most of the observed ${ }^{184 m 2} \mathrm{Hf}$ losses can be attributed to $\beta$ decay, rather than atomic electron capture. Taking into account that 15 additional ions were observed for one minute each, with one being lost from the ring, there were just five losses during 1.4 hours of observation time, and the nuclear decay mean-life can thus be estimated to be 24 minutes. After correcting for a Lorentz factor of $1.4, T_{1 / 2}=12_{-4}^{+10}$ minutes is obtained. (The uncertainties are evaluated according to the method given by Schmidt et al. [26].)

The remaining data have been analysed in a similar manner, although extended observations were not made for the other isomers. Isomer-to-ground-state transitions were observed from the first isomers of ${ }^{183,184} \mathrm{Hf}$ and ${ }^{186,187} \mathrm{Ta}$, implying $\gamma$-ray emission. An example of this is illustrated in Fig. 2, where it is seen that the trace corresponding to a single bare ion of ${ }^{187 m 1} \mathrm{Ta}$ terminates at $T \approx 48 \mathrm{~s}$, and is replaced by a trace corresponding to ${ }^{187 g} \mathrm{Ta}$. Only $\gamma$-ray emission(s) can give this behavior. Note that electron conversion is impossible from bare ions, and the neutral atoms are therefore likely to be shorter lived [27]. The detailed findings are given in Table I, where, of the seven isomers listed, only the first isomers of ${ }^{184} \mathrm{Hf}$ and ${ }^{186} \mathrm{Ta}$ had previously been reported $[14,28]$. In the latter case, the excitation energy was unknown. It has now been determined as $0.336(20) \mathrm{MeV}$, and we also find $\gamma$-decay events in addition to the reported $\beta$ decay [28]. The most exotic nuclide in which an isomer has been identified in the present work is ${ }^{186} \mathrm{Hf}$, where only two ions of the isomer were observed. Confirmation of this isomer, and the possibility to observe ${ }^{188} \mathrm{Hf}$ and its isomeric states, requires a beam that is more neutron-rich than ${ }^{197} \mathrm{Au}$, such as ${ }^{208} \mathrm{~Pb}$, as planned for a future experiment.

The theoretical understanding of the new isomers is based on the calculation of multi- 
quasiparticle states, using Nilsson-model single-particle energies and Lipkin-Nogami pairing, including blocking and residual interactions, according to the methodology of refs. [29, 30] with standard parameters. The predicted quadrupole, $\epsilon_{2}$, and hexadecapole, $\epsilon_{4}$, shape parameters were taken from Möller et al. [31]. For ${ }^{184} \mathrm{Hf}$, the $K^{\pi}=8^{-}$isomer at $1.272(1)$ $\mathrm{MeV}$ almost certainly has a two-quasi-proton $\left\{7 / 2^{+}[404] \otimes 9 / 2^{-}[514]\right\}$ configuration [14] (consistent with the lighter even-even hafnium isotopes). This is close to the calculated energy of $1.241 \mathrm{MeV}$ for that configuration. The clear candidate for the second ${ }^{184} \mathrm{Hf}$ isomer is a four-quasiparticle $K^{\pi}=15^{+}$state with the configuration $\pi^{2}\left\{7 / 2^{+}[404], 9 / 2^{-}[514]\right\} \otimes$ $\nu^{2}\left\{3 / 2^{-}[512], 11 / 2^{+}[615]\right\}$, calculated at $2.369 \mathrm{MeV}$, which matches well with the measured energy of $2.477(10) \mathrm{MeV}$.

In addition, the decay properties support this interpretation. The $K^{\pi}=15^{+}$isomer might be expected to $\gamma$ decay to a member of the rotational band built on the $K^{\pi}=8^{-}$ isomer. Using as a guide the energy spacings in the $K^{\pi}=8^{-}$bands observed in ${ }^{180} \mathrm{Hf}$ and ${ }^{182} \mathrm{Hf}$ [15], a $\sim 20 \mathrm{keV}, K$-forbidden $E 3$ transition is estimated for the $\gamma$ decay of the higher isomer in ${ }^{184} \mathrm{Hf}$. This would lead [8] to a partial $\gamma$-ray half-life that is much greater than the measured value of 12 minutes, consistent with the lack of observed $\gamma$-decay events. The measured half-life comes from $\beta$ decays to high-spin states in ${ }^{184} \mathrm{Ta}$.

Similar considerations can be used to explain the other newly observed isomers. For ${ }^{183} \mathrm{Hf}$ the calculations predict an energetically favored $K^{\pi}=27 / 2^{-}, \pi^{2}\left\{7 / 2^{+}[404], 9 / 2^{-}[514]\right\} \otimes$ $\nu\left\{11 / 2^{+}[615]\right\}$, three-quasiparticle state at $1.712 \mathrm{MeV}$, in reasonable agreement with the measured isomer at $1.464(64) \mathrm{MeV}$. For ${ }^{187} \mathrm{Ta}$, a good understanding of the isomers requires adjustment of the Nilsson energies to better match the known neutron levels in ${ }^{187} \mathrm{~W}[32]$. The calculations then favor a $K^{\pi}=41 / 2^{+}$, five-quasiparticle assignment for the upper isomer, with the configuration $\pi^{3}\left\{7 / 2^{+}[404], 9 / 2^{-}[514], 5 / 2^{+}[402]\right\} \otimes \nu^{2}\left\{9 / 2^{-}[505], 11 / 2^{+}[615]\right\}$. The calculated excitation energy of $2.710 \mathrm{MeV}$ is comparable to the experimental value of 2.935(14) MeV. A notable feature is that this isomer is longer lived ( $>5$ minutes) than the ground state (2.3 minutes). The lower isomer at $1.789(13) \mathrm{MeV}$ can be associated with a calculated $K^{\pi}=27 / 2^{+}, \pi\left\{9 / 2^{-}[514]\right\} \nu^{2}\left\{7 / 2^{-}[503], 11 / 2^{+}[615]\right\}$ three-quasiparticle state at $1.508 \mathrm{MeV}$. For ${ }^{186} \mathrm{Hf}$, a $K^{\pi}=17^{+}, \pi^{2}\left\{7 / 2^{+}[404], 9 / 2^{-}[514]\right\} \otimes \nu^{2}\left\{7 / 2^{-}[503], 11 / 2^{-}[615]\right\}$ four-quasiparticle state is calculated at $2.269 \mathrm{MeV}$ (without Nilsson energy adjustment) which is tentatively associated with the measured isomer at 2.968(43) MeV. Finally, for ${ }^{186} \mathrm{Ta}$ there are several two-quasiparticle states that are close in energy and could lead to 
isomer formation, but there is insufficient information to distinguish between the possibilities. Overall, it is found that the calculations provide an essential guide for interpreting the multi-quasiparticle isomer configurations and excitation energies. Nevertheless, further work is needed to understand the details of the single-particle energies, especially as the addition of further neutrons may lead to substantial shape changes [33].

In summary, storage-ring experiments have revealed highly excited, long-lived isomers in the neutron-rich $A \approx 180-190$ region of deformed nuclei, supporting earlier predictions. Now that the energies and half-lives are known, the way is open for the experimental design of detailed spectroscopic measurements.

The authors are grateful to the accelerator team for their excellent contribution. Geirr Sletten is thanked for his early involvement with the plans for these measurements. This work has been supported by the UK STFC, AWE plc, and DTRA (YSU).

[1] P.M. Walker, and G.D. Dracoulis, Nature 399, 35 (1999).

[2] F. Soddy, Nature 99, 433 (1917).

[3] P.M. Walker, and J.J. Carroll, Physics Today, 39, June 2005.

[4] F.R. Xu, E.G. Zhao, R. Wyss, and P.M. Walker, Phys. Rev. Lett. 92, 252501 (2004).

[5] S. Ćwiok, P.-H. Heenen, and W. Nazarewicz, Nature 433, 705 (2005).

[6] S. Åberg, Nucl. Phys. A 306, 89 (1978).

[7] F.R. Xu, P.M. Walker, and R. Wyss, Phys. Rev. C 62, 014301 (2000).

[8] P.M. Walker, and G.D. Dracoulis, Hyp. Int. 135, 83 (2001).

[9] G. Audi, O. Bersillon, J. Blachot and A. H. Wapstra, Nucl. Phys. A 729, 3 (2003).

[10] C.B. Collins et al., Phys. Rev. Lett. 82, 695 (1999).

[11] I. Ahmad et al., Phys. Rev. C 71, 024311 (2005).

[12] J.J. Carroll et al., Phys. Lett. B 679, 203 (2009).

[13] H. Grawe, K. Langanke, and G. Martinez-Pinedo, Rep. Prog. Phys. 70, 1525 (2007).

[14] K. Krumbholz et al., Z. Phys. A 351, 11 (1995).

[15] R. D'Alarcao et al., Phys. Rev. C 59, R1227 (1999).

[16] G.J. Lane et al., Phys. Rev. C 80, 024321 (2009).

[17] Zs. Podolyák et al., Phys. Lett. B 491, 225 (2000). 
[18] M. Caamaño et al., Eur. Phys. J. A 23, 201 (2005).

[19] N. Alkhomashi et al., Phys. Rev. C 80, 064308 (2009).

[20] Zs. Podolyák, et al., Phys. Lett. B 672, 116 (2009).

[21] H. Geissel et al., Nucl. Inst. Meth. Phys. Res. B 70, 286 (1992).

[22] B. Franzke, Nucl. Inst. Meth. Phys. Res. B 24/25, 18 (1987).

[23] B. Franzke, H. Geissel, and G. Münzenberg, Mass Spectrometry Reviews 27, 428 (2008).

[24] Yu. A. Litvinov, et al., Nucl. Phys. A 756, 3 (2005).

[25] B. Sun et al., Eur. Phys. J. A 31, 393 (2007).

[26] K.-H. Schmidt, C.-C. Sahm, K. Pielenz, and H.-G. Clerc, Zeit. Phys. A 316, 19 (1984).

[27] Yu.A. Litvinov et al., Phys. Lett. B 573, 80 (2003).

[28] Y. Xu et al., J. Phys. Soc. Japan 73, 2588 (2004).

[29] K. Jain, O. Burglin, G.D. Dracoulis, B. Fabricius, and P.M. Walker, Nucl. Phys. A 591, 61 (1995).

[30] F.G. Kondev, G.D. Dracoulis, A.P. Byrne, T. Kibédi, and S. Bayer, Nucl. Phys. A 617, 91 (1997).

[31] P. Möller, R.J. Nix, W.D. Myers and W.J. Swiatecki, At. Data Nucl. Data Tables 59, 185 (1995).

[32] M.S. Basunia, Nucl. Data Sheets 110, 999 (2009).

[33] L.M. Robledo, R. Rodríguez, and P. Sarriguren, J. Phys. G 36, 115104 (2009). 
TABLE I: Hafnium and tantalum isomers and ground states

\begin{tabular}{|c|c|c|c|c|c|c|c|}
\hline $\begin{array}{l}\text { Ion } \\
(q)\end{array}$ & level & $\begin{array}{l}\text { No. } \\
\text { ions }\end{array}$ & $\begin{array}{l}E_{E S R} \\
(\mathrm{MeV})\end{array}$ & $\begin{array}{l}E_{\text {calc }} \\
(\mathrm{MeV})\end{array}$ & $K_{\text {calc }}^{\pi}$ & $T_{1 / 2}{ }^{a}$ & $\begin{array}{l}\text { decay } \\
\text { mode }\end{array}$ \\
\hline${ }^{183} \mathrm{Hf}$ & $\mathrm{g}$ & 30 & 0 & 0 & $3 / 2^{-}$ & $1.07(2) \mathrm{h}^{b}$ & $\beta$ \\
\hline$(71+)$ & $\mathrm{m}$ & 1 & $1.464(64)$ & 1.712 & $27 / 2^{-}$ & $10_{-5}^{+48} \mathrm{~s}$ & $\gamma$ \\
\hline${ }^{184} \mathrm{Hf}$ & $\mathrm{g}$ & 86 & 0 & 0 & $0^{+}$ & $4.12(5) \mathrm{h}^{b}$ & $\beta$ \\
\hline \multirow[t]{2}{*}{$(72+)$} & $\mathrm{m} 1$ & 32 & $1.264(10)^{c}$ & 1.241 & $8^{-}$ & $113_{-40}^{+74} \mathrm{~s}^{c}$ & $\beta, \gamma$ \\
\hline & $\mathrm{m} 2$ & 20 & $2.477(10)$ & 2.369 & $15^{+}$ & $12_{-4}^{+10} \mathrm{~m}$ & $\beta$ \\
\hline${ }^{186} \mathrm{Hf}$ & g & 8 & 0 & 0 & $0^{+}$ & $2.6(12) \mathrm{m}^{b}$ & $\beta$ \\
\hline$(72+)$ & $\mathrm{m}$ & 2 & $2.968(43)$ & 2.269 & $17^{+}$ & $>20 \mathrm{~s}$ & \\
\hline${ }^{186} \mathrm{Ta}$ & $\mathrm{g}$ & 120 & 0 & & & $>10 \mathrm{~m}^{d}$ & $\beta$ \\
\hline$(72+)$ & $\mathrm{m} 1$ & 60 & $0.336(20)$ & & & $3.4_{-1.4}^{+2.4} \mathrm{~m}^{e}$ & $\beta, \gamma$ \\
\hline${ }^{187} \mathrm{Ta}$ & g & 102 & 0 & 0 & $7 / 2^{+}$ & $2.3(6) \mathrm{m}$ & $\beta$ \\
\hline \multirow[t]{2}{*}{$(73+)$} & $\mathrm{m} 1$ & 17 & $1.789(13)$ & $1.508^{f}$ & $27 / 2^{-}$ & $22(9) \mathrm{s}$ & $\beta, \gamma$ \\
\hline & $\mathrm{m} 2$ & 9 & $2.935(14)$ & $2.710^{f}$ & $41 / 2^{+}$ & $>5 \mathrm{~m}$ & \\
\hline
\end{tabular}

${ }^{a}$ Literature half-lives are for neutral atoms. The present values for isomers will be longer, because internal conversion is hindered or absent [27].

${ }^{b}$ From Audi et al. [9].

${ }^{c}$ Consistent with previously measured $1.272(1) \mathrm{MeV}$ and $48(10) \mathrm{s}$ for the neutral atom $[9,14]$, after correcting for internal conversion.

${ }^{d}$ cf. 10.5(3) m from Audi et al. [9].

${ }^{e}$ cf. 1.54(5) $\mathrm{m}$ reported for the neutral atom [28].

${ }^{f}$ After adjustment of Nilsson levels to reproduce ${ }^{187} \mathrm{~W}$ (see text). 


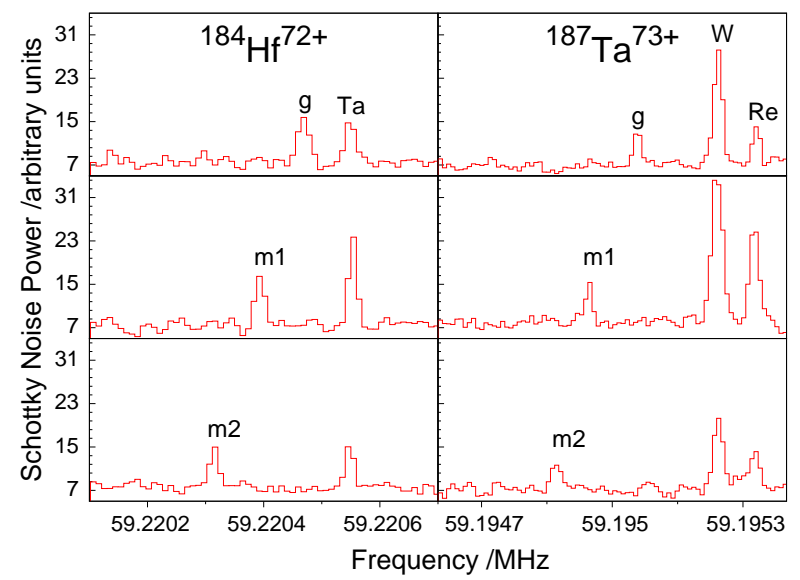

FIG. 1: (Color online) Examples of $30^{\text {th }}$ harmonic Schottky spectra for $A=184$ (left) and 187 (right) isobars and isomers, each representing $10 \mathrm{~s}$ of data, with different distributions of ions from different injections in the ESR. The ${ }^{184} \mathrm{Hf}$ and ${ }^{187} \mathrm{Ta}$ isomers ( $\mathrm{m} 1$ and $\left.\mathrm{m} 2\right)$ and ground states $(g)$ are identified as individual bare ions. The ground states of ${ }^{184} \mathrm{Ta}^{72+},{ }^{187} \mathrm{~W}^{73+}$ and ${ }^{187} \mathrm{Re}^{73+}$ are also indicated. The statistical significance of single-ion identifications is $5 \sigma$ for $10 \mathrm{~s}$ of observation time (as illustrated) and greater than $5 \sigma$ for the typical $40 \mathrm{~s}$ of observation per injection. The total numbers of observed ions are given in Table I. 


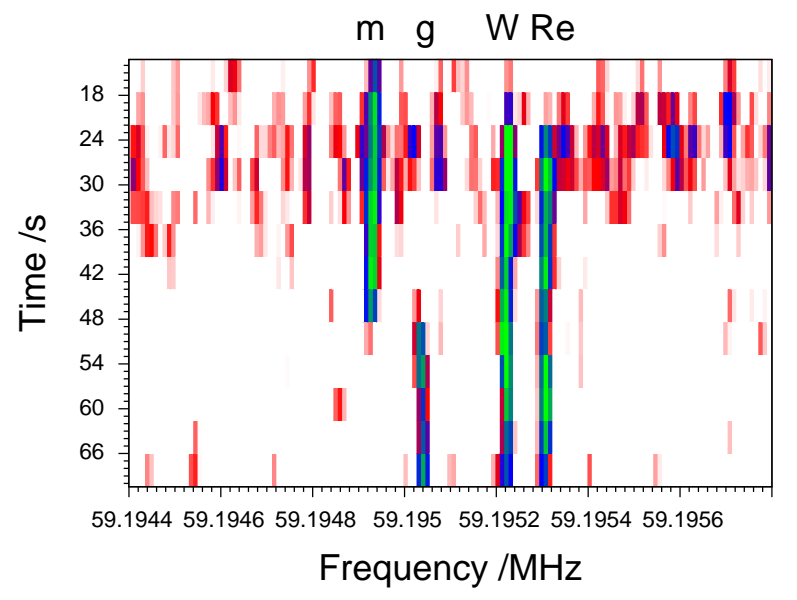

FIG. 2: (Color online) Time evolution of $A=187, q=73+$ Schottky spectra for a single injection in the ESR with 4.4-s binning. During the early times there is cooling. The left-hand vertical trace $(\mathrm{m})$ is for a single bare ion of the first isomer of ${ }^{187} \mathrm{Ta}$. After $48 \mathrm{~s}$ it $\gamma$ decays, represented by a sudden increase in frequency, to the ${ }^{187}$ Ta ground state $(g)$. The two uninterrupted vertical traces are for the ${ }^{187} \mathrm{~W}$ and ${ }^{187}$ Re ground states (cf. Fig. 1). 